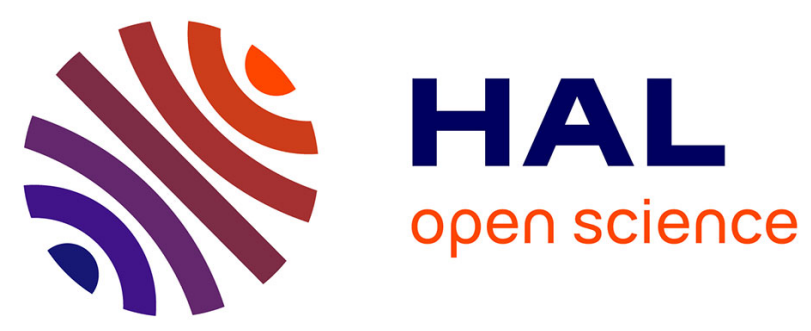

\title{
SATELLITE IMAGE RECONSTRUCTION FROM AN IRREGULAR SAMPLING
}

Eric Bughin, Laure Blanc-Féraud, Josiane Zerubia

\section{To cite this version:}

Eric Bughin, Laure Blanc-Féraud, Josiane Zerubia. SATELLITE IMAGE RECONSTRUCTION FROM AN IRREGULAR SAMPLING. ICASSP, Mar 2008, Las Vegas, NEVADA, United States. inria-00278919

\section{HAL Id: inria-00278919 \\ https://hal.inria.fr/inria-00278919}

Submitted on 14 May 2008

HAL is a multi-disciplinary open access archive for the deposit and dissemination of scientific research documents, whether they are published or not. The documents may come from teaching and research institutions in France or abroad, or from public or private research centers.
L'archive ouverte pluridisciplinaire HAL, est destinée au dépôt et à la diffusion de documents scientifiques de niveau recherche, publiés ou non, émanant des établissements d'enseignement et de recherche français ou étrangers, des laboratoires publics ou privés. 


\title{
SATELLITE IMAGE RECONSTRUCTION FROM AN IRREGULAR SAMPLING
}

\author{
Eric Bughin, Laure Blanc-Féraud, Josiane Zerubia \\ Projet Ariana - CNRS/INRIA/UNSA \\ firstname.lastname@inria.fr \\ INRIA Sophia-Antipolis \\ 2004, route des Lucioles, BP 93 \\ 06902 Sophia-Antipolis - FRANCE
}

\begin{abstract}
We propose a new method to solve a problem of image restoration with many different aspects: reconstruction from irregular samples, deconvolution and denoising. The model we propose is robust to different kind of noises, in particular, impulse and Gaussian noise. We compare our results to the ones obtained in [1] and show that our problem presents some advantages particularly in satellite imaging. At last, we conclude on a discussion about resolution schemes for variational problems' minimization and propose some faster resolution shemes for our problem and the one in [1].
\end{abstract}

Index Terms - Irregular sampling, Variational methods, Fourier Analysis, Satellite imaging.

\section{INTRODUCTION}

The problem of reconstructing an image from a random set of irregular samples has been fewly explored and becomes a problem of great interest in various domain such as biomedical imaging or satellite imaging. The whole difficulty of such a problem is to find a method to restore a regularly sampled image from its irregular samples knowing the shifts between the irregular grid and the regular grid. Hence, the problem can be seen as finding the inverse of a regular to irregular sampling operator. The difficulty is that the positions of the irregular samples are totally arbitrary and such a system may not be invertible.

Various method have been developed, in particular exact reconstruction methods ([2], [3]) which require large sets of data without any noise, and variational methods ([4], [1]) that require more computation time but are more adapted to noisy data. Moreover, using a smoothing term will allow a reconstruction from an irregular sampling which can be sparse in some places and very dense elsewhere. We propose a new

The authors would like to thank the Compagny CS in Toulouse (France) for partial funding and the Space French Agency CNES for providing the data. We particularly thank Anne Chanié and Pauline Audenino for several interesting discussions. variational approach to restore satellite images from an irregular sampling and propose to compare it to the method proposed in [1]. At last, in order to generate an irregular sampling, we used satellite stereoscopic images.

Let's consider a satellite stereoscopic acquisition of a scene. Then we have two regular acquisition of the same scene. By applying the disparities between the two image to the reference image, we get an irregularly sampled new image which should be identical to the second image of the stereopsis pair (appart from some details due to moving objects during the time between the acquisitions of the stereoscopic pair). As a matter of fact, the second image can be considered as an irregularly sampled acquisition (in comparison to the reference image) and the problem of reconstructing the reference image from the second image knowing the disparities between the two images can be considered as an irregular sampling problem. A general acquisition model can be described as follows:

$$
u=\Delta_{\Lambda} \cdot\left(u_{0} * h\right)+n
$$

where $u_{0}$ is the scene that we want to acquire, $h$ is a convolution kernel, for instance the PSF (Point Spread Function) of the acquisition system of the satellite, $\Delta_{\Lambda}$ is an irregular sampling of the scene which can be seen as a sum of Dirac functions centered at the irregular samples positions:

$$
\Delta_{\Lambda}(.)=\sum_{\lambda_{k} \in \Lambda} \delta\left(.-\lambda_{k}\right)
$$

At last, the noise $n$ that we consider for our model has two different aspects: a first part of the noise is due to the acquisition system and can be considered as a white Gaussian noise, a second part of the noise can be due to errors in the computation of the disparities between the two images of the stereoscopic pair. Such an error may have disastrous effects in urban images: a bad estimation in the position of a sample located on the top of building (let's suppose it as a white pixel), may place this sample in a place where there should be some shadow (black pixel). As a matter of fact, some errors in the estimation of irregular samples may completely change the value of a pixel, which can be seen as an impulse noise. 
Based on the problem proposed in [1] by Almansa et al, and motivated by the previous discussion, we propose to minimize the following problem:

$$
\left\|\Delta_{\Lambda} \cdot(u * h)-g\right\|_{1}+\lambda J(u)
$$

where $u$ is the regular image that we want to reconstruct, $g$ are the irregular samples, $J(u)$ is the total variation of $u$ defined by $J(u)=\int|\nabla u|$ and $\lambda$ a parameter that weights the regularisation of the solution by the total variation. The choice of such an approach is justified by three criterion:

- a variational approach will be robust to Gaussian noise. Even if we use an $\ell_{1}$ norm on the data fidelity term, when the variance of the Gaussian noise is not too strong (which is the case in satellite imaging), the smoothing due to the total variation is enough to denoise the image.

- a regularisation by total variation will keep the high gradient zone of the image in place.

- the use of an $L_{1}$ norm on the data fidelity term will be robust to impulse noise.

\section{DISCRETIZED PROBLEM}

In its discrete form, the problem can be matricially defined by:

$$
\|S H F u-g\|_{1}+\lambda J(u)
$$

Where $\|.\|_{1}$ is the $\ell_{1}$ norm, $F$ is the discrete fast Fourier transform, $H$ is the Fourier transform of the $P S F h$ of the satellite, $S$ is a transform that create an irregularly sampled image from its regular samples in the Fourier domain. This last operator is described in [3] and [1] and can be fastly computed (the direct computation is in $\mathcal{O}\left(N^{2}\right)$ ) with the USFFT (Unequally Spaced Fast Fourier Transform) developed by G. Beylkin in [5]. For simplicity reasons, let us note $A$ the operator defined by $A=S H F$.

The problem of efficiently minimizing an $\ell_{1}-\ell_{1}$ energy function such as the one we propose to solve is a difficult problem. In order to experimentally show the validity of our model, we propose to use a gradient descent to minimize the energy function. As the $\ell_{1}$ norm is not $C_{1}$ in 0 , we propose to regularize the $\ell_{1}$, norm with a parameter $\mu$ :

$$
\|u\|_{1}=\sum_{k}\left|u_{k}\right| \stackrel{\mu}{\longrightarrow} \quad\|u\|_{1, \mu}=\sum_{k} \sqrt{\left|u_{k}\right|^{2}+\mu^{2}}
$$

We finally obtain the following gradient descent:

$$
\begin{aligned}
u_{n+1}=u_{n}-\tau & \left(A^{*}\left(\frac{A u_{n}-g}{\sqrt{\left|A u_{n}-g\right|^{2}+\mu^{2}}}\right)\right. \\
& \left.-\lambda \operatorname{div}\left(\frac{\nabla u_{n}}{\sqrt{\left|\nabla u_{n}\right|^{2}+\mu^{2}}}\right)\right)
\end{aligned}
$$

Where $A^{*}$ is the adjoint operator of $A, \tau$ is the gradient descent step and must respect the inequality $\tau<2 / L$, with $L$ the Lipschitz constant of the problem defined by:

$$
L=\frac{\|A\|_{2}^{2}+\lambda\|\operatorname{div}\|_{2}^{2}}{\mu}
$$

The choice of the parameter $\mu$ is very important for two reasons:

- the descent gradient step is directly proportional to $\mu$. Hence, the bigger $\mu$ is, the bigger the gradient descent step is and the faster the gradient descent will converge.

- A too strong parameter $\mu$ causes a bad approximation of the $\ell_{1}$ norm. Hence, the result might become blurry with a big parameter $\mu$.

In practice we chose the $\mu$ parameter experimentally. Once chosen, this parameter is the same from an image to another if the two images have the same dynamic.

\section{RESULTS}

We have tested our algorithm on different images irregularly sampled. The results seems to be good after our regular resampling (in comparison to the reference image). The method proposed by Almansa et al gives the same results in terms of registration. It should be noted that for images without any noise, a direct reconstruction with a good interpolation gives the similar result. Nevertheless, as we are dealing with satellite images, our method has to be robust to additive white Gaussian noise. In order to test the validity of the registration, we have subtracted the result obtained with our algorithm to the reference image. By visualizing the histogram of these images of differences, it can be seen that the variance is very low (the standard deviation is equal to 2 ) after the application of our algorithm (the mean value is 0 ). This means that our reconstruction algorithm gives a result near from the reference image (regularly sampled).

We have tested our reconstruction algorithm on images irregularly sampled with a signal to noise ratio $S N R=15.5$ $\mathrm{dB}$ (for a white Gaussian noise). We recall that the signal to noise ratio is given by the following formula:

$$
S N R=20 \log \frac{\sigma_{\text {signal }}}{\sigma_{\text {noise }}}
$$

For this kind of noise, we obtain a reconstruction (regular resampling + denoising) as good as the one that is obtained in [1]. For images with a much lower signal to noise ratio (noise with a larger variance), Almansa et al obtain a better result with a low regularization, and the same result with a large regularization. These results are due to the fact that the $\ell_{2}$ norm (on the data fidelity term) is more robust to the white Gaussian noise than the $\ell_{1}$ norm. Nevertheless in practice, the noise due to satellite sensors is not large enough to observe 
much difference between the two methods.

As it was said before, it is interesting for our algorithm to be robust to impulse noise. We have tested the quality of reconstruction with noisy images with $10 \%$ of impulse noise. Contrarily to $\ell_{2}-\ell_{1}$ methods like the one proposed in [1], our method proved itself to be robust to this kind of noise. An $\ell_{2}-\ell_{1}$ method will need a stronger smoothing with the total variation to get rid of the same noise. The result of impulse noise denoising is given on figure $1^{1}$.
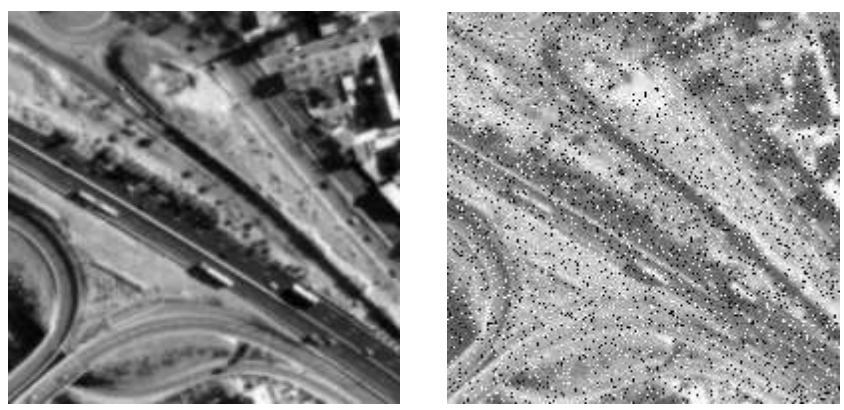

(a)

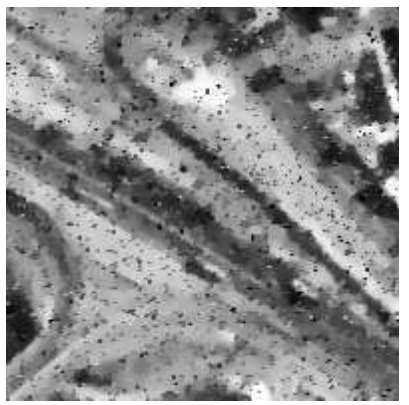

(c)

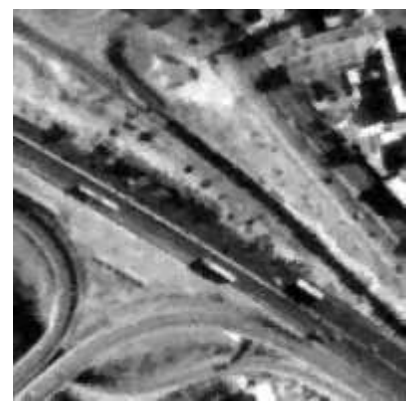

(d)

Fig. 1. Robustness to noise: (a) Reference Image, (b) input (irregularly sampled) with additive Gaussian noise

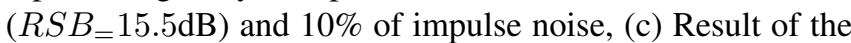
algorithm of Almansa et al (regular sampling + denoising), (d) Result of our algorithm (regular sampling + denoising)

\section{DISCUSSION AND CONCLUSION}

We have proposed a novel method to reconstruct satellite images from an irregular sampling knowing the position of

\footnotetext{
${ }^{1}$ Thanks to the CNES agency for allowing us to use their images.
}

the samples. This method includes a deconvolution by the $P S F$ of the satellite and was proved to be robust to two different kind of noises: the Gaussian noise due to satellite sensor, the impulse noise which can occur with errors on the estimation of the position of the samples. Our problem is based on minimizing the energy function given in (2) which consist on a $\ell_{1}$ norm for the data fidelity term and an $\ell_{1}$ norm for the regularization term (total variation). Minizing such an energy function is called an $\ell_{1}-\ell_{1}$ problem. Moreover, we compared our method to the one proposed by Almansa et al in [1] which is an $\ell_{2}-\ell_{1}$ problem problem $\left(\ell_{2}\right.$ norm for the data fidelity term).

The problem of minimzing an $\ell_{1}-\ell_{1}$ problem is that the convergence of an iterative algorithm such as the gradient descent require more iteration in comparison to $\ell_{2}-\ell_{1}$ problems. Moreover, dual approaches (for instance Chambolle algorithm [6]) can be used with $\ell_{2}-\ell_{1}$ methods and it is also much more easier to use an accelerated scheme such as Nesterov's one [7] [8] [9](for dual and primal problems). These last resolution schemes can considerably reduce the number of iterations to the convergence and make $\ell_{2}-\ell_{1}$ methods much more attractive than $\ell_{1}-\ell_{1}$. However, to be in the right configuration for applying Nesterov's algorithm on a problem using an operator $A$, it is either necessary to know how to do a projection with a convolution with $A$, or to be able to compute the inverse of the operator $A$. As our case, the operator $A$ depends on the sampling, it may not be invertible. Another solution for the $\ell_{2}-\ell_{1}$ problem could be to use the Prox functions ([10]) with the following scheme:

$$
\begin{gathered}
u_{n+1}=\operatorname{Prox}_{\gamma}\left(u_{n}-\gamma \lambda A^{*}\left(A u_{n}-g\right)\right) \\
\operatorname{Prox}_{\gamma}(x)=\inf _{u}\left(J(u)+\gamma\|u-x\|_{2}^{2}\right)
\end{gathered}
$$

The Prox step can then be efficiently solved using the dual Nesterov algorithm and the computation of the inverse matrix of $A$ is not necessary any more. In [11], Bect et al obtained the same kind of algorithm. A nice solution to $\ell_{1}-\ell_{1}$ problems was proposed by $\mathrm{Fu}$ et al in [12]. The idea is to reformulate a problem with a non-negativity constraint under the form of a linear programming problem. If we consider the following problem:

$$
\min _{u}\|A u-g\|_{1}+\alpha\|R u\|_{1}
$$

Where $R$ is a regularisation function (if $R$ is the first order difference operator, then $\|R u\|_{1}$ is the total variation of $u$ ). Let $v=A u-g$ and $w=\alpha R u$. $v$ and $w$ can be reformulated with their non-negative and non-positive part. Hence, we have $v=v^{+}-v^{-}$and $w=w^{+}-w^{-}$where $v^{+}=\max (v, 0), v^{-}=\max (-v, 0), w^{+}=\max (w, 0)$ and at last $w^{-}=\max (-w, 0)$. The problem (10) can now be reformulated as:

$$
\min _{u, v^{+}, v^{-}, w^{+}, w^{-}} \mathbf{1}^{T} v^{+}+\mathbf{1}^{T} v^{-}+\mathbf{1}^{T} w^{+}+\mathbf{1}^{T} w^{-}
$$


With the constraints:

$$
\left\{\begin{array}{l}
A u-g=v^{+}-v^{-} \\
\lambda R u=w^{+}+w^{-} \\
u, v^{+}, v^{-}, w^{+}, w^{-} \geq 0
\end{array}\right.
$$

At last, (11) can be written as the following linear programing problem:

$$
\min _{\mathbf{x}} \mathbf{c}^{T} \mathbf{x} \quad \text { with the constraints } \quad \mathbf{T} \mathbf{x}=\mathbf{b}, \mathbf{x} \geq 0
$$

Where $\mathbf{c}, \mathbf{T}, \mathbf{x}$, and $\mathbf{b}$ are defined by:

$$
\begin{array}{ccc}
\mathbf{T}=\left(\begin{array}{ccccc}
A & -I & I & 0 & 0 \\
\alpha R & 0 & 0 & -I & I
\end{array}\right), \quad \mathbf{b}=\left(\begin{array}{l}
g \\
0
\end{array}\right) \\
\mathbf{x}=\left(\begin{array}{c}
u \\
v^{+} \\
v^{-} \\
w^{+} \\
w^{-}
\end{array}\right) & \mathbf{c}=\left(\begin{array}{l}
0 \\
\mathbf{1} \\
\mathbf{1} \\
\mathbf{1} \\
\mathbf{1}
\end{array}\right)
\end{array}
$$

The Lagrangian function of (11) is:

$$
\mathcal{L}(\mathbf{x}, \lambda, \mathbf{s})=\mathbf{c}^{T} \mathbf{x}-\lambda(\mathbf{T} \mathbf{x}-\mathbf{b})-\mathbf{s}^{T} \mathbf{x}
$$

Where $\lambda$ and $\mathbf{s}$ are respectively the Lagrange multipliers for $\mathbf{T} \mathbf{x}=\mathbf{b}$ and $\mathbf{x} \geq 0$.

Once the problem has been reformulated as a linear programing problem, many different schemes exist to solve it. One of the most popular, and the one used in [12] is the interior points method (in [12], the authors combine the interior points method with conjugate gradients). In comparison to a gradient descent, this new scheme is a lot faster, and using an $\ell_{1}-\ell_{1}$ method becomes of great interest.

\section{REFERENCES}

[1] Andrés Almansa, Vicent Caselles, Gloria Haro, and Bernard Rougé, "Restoration and zoom of irregularly sampled, blurred, and noisy images by accurate total variation minimization with local constraints," Multiscale Modeling \& Simulation, vol. 5, no. 1, pp. 235-272, 2006.

[2] Akram Aldroubi and Karlheinz Gröchenig, "Nonuniform sampling and reconstruction in shift-invariant spaces," SIAM Rev., vol. 43, no. 4, pp. 585-620, 2001.

[3] Hans G. Feichtinger, Karlheinz Gröchenig, and Thomas Strohmer, "Efficient numerical methods in non-uniform sampling theory," Numerische Mathematik, vol. 69, pp. 423-440, 2000.

[4] Muthuvel Arigovindan, Michael Sühling, Patrick R. Hunziker, and Michael Unser, "Variational image reconstruction from arbitrarily spaced samples: a fast multiresolution spline solution.," IEEE Transactions on Image Processing, vol. 14, no. 4, pp. 450-460, 2005.
[5] Gregory Beylkin, "On the fast Fourier transform of function with singularities.," Applied and Computational Harmonic Analysis, vol. 2, no. 4, pp. 363-381, 1995.

[6] Antonin Chambolle, "An algorithm for total variation minimization and applications," J. Math. Imaging Vis., vol. 20, no. 1-2, pp. 89-97, 2004.

[7] Yurii Nesterov, "A method for unconstrained convex minimization problem with the rate of convergence $O\left(\varepsilon^{-2}\right), "$ Doklady AN SSSR, vol. 269, no. 3, pp. 543$547,1983$.

[8] Yurii Nesterov, "Smooth minimization of non-smooth functions," Mathematic Programming, Ser. A, vol. 103, pp. 127-152, 2005.

[9] Pierre Weiss, Gilles Aubert, and Laure Blanc-Féraud, "Efficient schemes for total variation minimization under some convex constraints in image processing," Tech. Rep., INRIA, 2007.

[10] P. L. Combettes and V. R. Wajs, "Signal recovery by proximal forward-backward splitting," SIAM Journal on Multiscale Modeling and Simulation, vol. 4, no. 4, pp. 1168-1200, 2005.

[11] J. Bect, L. Blanc-Féraud, G. Aubert, and A. Chambolle, "A $l^{1}$-unified variational framework for image restoration," in Proc. European Conference on Computer Vision (ECCV), T. Pajdla and J. Matas, Eds., Prague, Czech Republic, mai 2004, vol. LNCS 3024, pp. 1-13, Springer.

[12] H. Haoying Fu, M. Ng, M. Nikolova, and J. Barlow, "Efficient minimization methods of mixed 11-11 and 12-11 norms for image restoration," SIAM Journal on Scientific computing, vol. 27, no. 6, 2006. 